\title{
Conhecendo a Comunidade de Sistemas de Informação no Brasil: um Estudo Comparativo Utilizando Diferentes Abordagens de Banco de Dados
}

\author{
Alternative Title: Knowing the Brazilian Information Systems Community: A \\ Comparative Study with Different Database Approaches
}

\author{
Natan de S. Rodrigues \\ Departamento de Ciência da Computação \\ Instituto de Ciências Exatas \\ Universidade de Brasília \\ Brasília - DF - Brasil \\ natan@aluno.unb.br
}

\author{
Célia G. Ralha \\ Departamento de Ciência da Computação \\ Instituto de Ciências Exatas \\ Universidade de Brasília \\ Caixa Postal 4466 - Brasília - DF \\ CEP 70.904-970 \\ ghedini@cic.unb.br
}

\begin{abstract}
RESUMO
Este artigo apresenta o resultado de uma pesquisa que tem como objetivo disponibilizar informações sobre a comunidade de Sistemas de Informação no Brasil, utilizando diferentes abordagens de banco de dados. O estudo de viabilização de informação utilizou como base os dados das publicações na trilha principal do Simpósio Brasileiro de Sistemas de Informação (SBSI) das edições de 2005 a 2014. Os dados das diversas edições do SBSI foram armazenados em dois bancos de dados construídos utilizando abordagens diferentes de banco de dados (BD). Foi feito também um estudo sobre aspectos de eficiência das abordagens utilizadas com a finalidade de investigar qual das alternativas traz melhor resultado para o armazenamento e a recuperação das informações sobre a comunidade.
\end{abstract}

\section{Palavras-Chave}

BD, Comunidade Científica, Rede Social, SBSI

\begin{abstract}
This paper presents the results of a research that has the objective to provide information about the Brazilian Information Systems Community. The study used data from the articles published on the main track of the Information Systems Brazilian Symposium (SBSI) from 2005 to 2014. The data were stored in two databases (DB), using different approaches database (relational and NoSQL).A study about efficiency aspects of both database approaches was conducted in order to investigate which alternative is better to store and retrieve information about the community.
\end{abstract}

Permission to make digital or hard copies of all or part of this work for personal or classroom use is granted without fee provided that copies are not made or distributed for profit or commercial advantage and that copies bear this notice and the full citation on the first page. To copy otherwise, to republish, to post on servers or to redistribute to lists, requires prior specific permission and/or a fee.

SBSI 2015, May 26th-29th, 2015, Goiânia, Goiás, Brazil

Copyright SBC 2015.

\section{Categories and Subject Descriptors}

H.4 [Information Systems Applications]: Miscellaneous; H.2.8 [Information Systems]: Database Applications-Scientific databases

\section{General Terms}

Análise dos dados, BD, SBSI

\section{Keywords}

DB, SBSI, Scientific Community, Social Network

\section{INTRODUÇÃO}

No avanço da tecnologia e na interação entre os computadores, a utilização de Sistemas de Informação (SI) é algo evidente e de grande valor, resultando com a popularização do tema e o crescimento de pesquisas que focam na área. Como em outros países, no Brasil atualmente existe uma rede de pesquisadores e pesquisas atuantes na Comunidade de SI, que tem crescido conforme pode ser observado nas publicações do Simpósio Brasileiro de Sistemas de Informação (SBSI).

O SBSI é um evento que ocorre anualmente tendo sido escolhido neste trabalho para representar a comunidade de pesquisadores de SI no Brasil, uma vez que é o principal evento da área. Para o estudo dessa comunidade, a necessidade de registrar em um base de dados as informações da comunidade, a fim de se conhecer os membros integrantes e poder tomar decisões que viabilizem o seu crescimento integrado e produtivo. Desta forma, foram elaboradas questões centrais para esta pesquisa, as quais serviram de base para direcionar os estudos da comunidade, a saber:

- Qual o volume de artigos aceitos nas diversas edições do SBSI?

- Quais os autores que mais publicam no SBSI e com qual frequência?

- Quais são as instituições de vinculação destes autores?

- Com quem estes autores publicam? 
Considera-se também importante identificar todos os autores que publicaram em co-autoria com determinado autor, durante todas as edições do SBSI realizadas.

Com a finalidade de responder as questões de pesquisa apresentadas percebeu-se a necessidade de desenvolver um $\mathrm{BD}$ com as diversas edições do SBSI, para recuperar e disponibilizar as informações da comunidade de SI do Brasil. Assim, podemos popular um BD e montar uma rede social de forma digital, disponibilizando de forma viável as informações sobre esta comunidade científica. Os dados necessários para alimentar o BD serão os artigos publicados no SBSI que estão disponibilizados nas Trilhas Técnicas anuais de cada evento. De forma a obter o melhor desempenho e eficiência sobre as disponibilização das informações acerca da rede social científica, tornou-se necessário o armazenamento dos dados da comunidade utilizando-se duas abordagens e estruturas distintas, pois assim é possível analisar e verificar qual a melhor alternativa para armazenamento de informações de uma rede social científica.

Assim, como objetivo deste trabalho temos a criação da base de dados utilizando duas abordagens distintas de BD, com as edições 2005, 2006, 2008, 2009, 2010, 2011, 2012, 2013, 2014 do SBSI, pois na base da SBC, o DBDComp, atualmente existem somente sete edições do SBSI (2008 a 2014) e analisar a eficiência dos bancos de dados utilizados. A base de dados criada neste trabalho está disponibilizada no sítio http://natanrodrigues.eti.br/sbsi, que ainda está em versão de testes. Através do sítio é possível recuperar informações de publicação na trilha principal do SBSI das edições 2005 a 2014 e baixar a base SQL e NoSQL para futuras pesquisas.

O restante do artigo está organizado da seguinte forma: na Seção 2 são apresentados os trabalhos correlatos; na Seção 3 discorre-se sobre os métodos e tecnologias utilizadas neste trabalho; na Seção 4 é detalhado a proposta metodológica utilizada; na Seção 5 a análise dos resultados é descrita; e na Seção 6 as conclusões e trabalhos futuros são discutidos.

\section{TRABALHOS CORRELATOS}

O estudo realizado em [6] e publicado em [1] serviu como base para a realização deste trabalho, pois foi utilizada a base de dados de 2006 a 2011 disponibilizada pelo autor. Em [1], os autores fizeram uma análise de colaboração entre os pesquisadores e instituições participantes do SBSI, utilizando o conceito de corte de vértices em grafos e análise de redes sociais. Os autores utilizaram uma base de dados relacional com as informações dos artigos, autores e instituições, juntamente com suas relações, aplicando algoritmos para possibilitar a análise da rede social com corte de vértices em grafos.

Algumas informações relevantes foram identificadas pelo autores. A pesquisa identificou que $25 \%$ das instituições não estabeleceram parcerias na publicação de artigos nas edições do SBSI de 2006 a 2011. Outra informação identificada foi que autores com destaque em determinadas edições do SBSI não apresentavam destaque em outras edições do SBSI.

O artigo [7], publicado na edição de 2014 do SBSI, também serviu de base para a elaboração deste trabalho. No artigo, os autores definem alguns critérios para a escolha da utilização em projetos de um BD NoSQL. Algumas características como, bom desempenho, maior grau de escalabilidade, flexibilidade e disponibilidade são atribuídas aos BD NoSQL. Assim definindo critérios que devem ser observados para a escolha desse tipo de $\mathrm{BD}$, auxiliando a escolha dessa abordagem utilizada neste trabalho.

Para análise e definição dos critérios para utilização de um BD NoSQL, os autores buscaram na literatura os principais critérios e validaram junto a um grupo de 32 especialistas do tema. Por fim, a pesquisa identificou 22 critérios relevantes que devem ser identificados pelas organizações ao utilizaram um BD NoSQL.

\section{MÉTODOS E TECNOLOGIAS}

Esta seção descreve os métodos e tecnologias utilizados neste trabalho. Serão identificados os conceitos de banco de dados relacional e banco de dados orientado a grafos.

\subsection{Abordagem Relacional}

O idealizador do modelo relacional define em seu estudo [4] que o banco de dados relacional armazena os dados utilizando tabelas bidimensionais. De acordo com [3], um banco de dados relacional é composto de tabelas. Uma tabela é um conjunto não ordenado de tuplas, que são compostas por uma série de atributos. O conjunto de atributos que possuem o mesmo nome formam uma coluna.

A manipulação de tabelas é feita com os dados alocados em tuplas e colunas, assim utilizando como ferramenta para manipulação a álgebra relacional, que conta com conjunto de operadores e funções de alto nível.

Algumas características e vantagens de se utilizar um banco de dados que utiliza uma abordagem relacional foram explicitadas pelos autores em [14]:

- Independência total dos dados;

- Visão múltiplas dos dados;

- Redução acentuada na atividade de desenvolvimento de aplicações e o tempo gasto em manutenção.

O sistema de gerenciamento de banco de dados relacional que será utilizado neste trabalho é o MySQL. De acordo com [15], o MySQL é um sistema de fácil manipulação e é funcional para aplicações na Web. A escolha desse Sistema de Gerenciamento de Banco de Dados (SGBD) para a confecção deste trabalho foi por ser um sistema multi-plataforma, ter compatibilidade com várias linguagens e ser um software livre com base na GNU General Public License (GPL) [9].

\subsection{Abordagem Orientada a Grafos}

De acordo com [18], no BD orientado a grafos existem as arestas (relacionamentos) e os vértices (nós), estruturas nas quais os dados são armazenados.

As estruturas utilizadas no BD Orientado a Grafos armazenam os dados, diferente do BD relacional que utiliza os relacionamentos apenas para referenciação de tabelas. Por exemplo: em uma família temos as pessoas e elas são ligadas pelo grau de parentesco, onde o grau de parentesco são os relacionamentos e as pessoas são os nós.

Segundo Moniruzzaman e Hossain [17], o BD orientado a grafos é mais útil quando a recuperação de informações dos relacionamentos é mais importante que propriamente os dados. Os autores também exemplificam vários tipos desse BD. Em [8], encontramos um detalhamento de cada BD Orientado a Grafos, onde são mostradas as características de cada um, incluindo os prós e contras de cada BD. 
De acordo com [10], o AllegroGraph é um BD Orientado a Grafos de alta performance, que utiliza a memória de forma eficiente juntamente com o armazenamento em disco, assim escalonando bilhões de quadros enquanto mantém a eficiente performance.

Segundo [13], O InfiniteGraph é um BD Orientado a Grafos. Uma das características do InfiniteGraph é a persistência dos dados utilizando relações complexas e múltiplos saltos no vasto e distribuído armazenamento de dados.

O Neo4j é um banco de dados orientado a grafos e desenvolvido pela empresa Neo Technology, sendo um dos mais referenciados na literatura [12]. O Neo4j é capaz de armazenar os dados em nós interligados por relacionamentos, assim BD complexos, pode ser manipulado com apenas uma instância do servidor. De acordo com [19], o BD orientado a grafos Neo4j pode ser utilizado para análise de uma rede social, modelando de forma natural uma rede, podendo também realizar persistências nesse BD.

Segundo [8], o Neo4j também conta com extensões e integrações com linguagens e frameworks, tais como Java, Ruby e Phyton, sendo algumas de suas API's encontradas no site desse banco de dados. De acordo com o autor [16], a estrutura do banco de dados Neo4j é composta por três elementos:

- Nó: corresponde ao vértice do modelo em grafos, cada nó tem um único ID;

- Relacionamento: serve para ligar dois nós, assim gerando um relacionamento entre os dois vértices;

- Propriedade: é um atributo que pode existir em um nó ou relacionamento, e.g., nome ou idade.

Após a análise dos tipos de BD orientado a grafos apresentados foi definido que a melhor opção a ser utilizada neste trabalho é o Neo4j, pois segundo [8], ele conta com extensões para várias linguagens e é um dos únicos que é open-source para fins não-comerciais.

\section{PROPOSTA METODOLÓGICA}

O SBSI é um evento realizado anualmente, que reune apresentações de trabalhos científicos e a discussão de temas relevantes da área de SI, aproximando pesquisadores, estudante e empresários. Para a confecção deste trabalho, foram utilizados os dados referentes aos simpósios realizados de 2006 a 2014, assim servindo para integrarem os BD's utilizados na pesquisa e obtendo uma rede social digital com as informações relacionadas às publicações dos eventos.

De acordo com [5] há métodos para se obter conceitos e padrões em estruturas de $\mathrm{BD}$, assim revelando relações entre as partes dessas estruturas. Segundo [5], uma mineração e análise de dados podem ser executadas em estruturas representadas por grafos, sendo assim possível uma análise social em uma rede social representada por grafos.

Com a finalidade de alcançar os objetivos proposto neste trabalho foi utilizada uma metodologia teórica e prática, que compreende desde o estudo de diferentes abordagens de BD, ferramentas e recursos adequados, até a definição de um modelo BD que utilize pelo menos duas abordagens diferentes para viabilizar a comparação de performance dos recursos utilizados.

Primeiramente, foram feitas as modelagens dos BD's. As características das modelagens são apresentadas nas Seções 4.1 e 4.2 .
Após a modelagem, os BD's foram criados e carregados com as informações dos artigos publicados nas edições do SBSI de 2006 a 2014, com os relacionamentos e modelagem adaptados para cada modelo de BD utilizado.

Após a construção dos BD's, foi feita a análise dos dados da rede social para obter as respostas das questões propostas na Seção 1. Em seguida, foram comparados os resultados de consultas aplicadas nesses modelos e assim realizada a experimentação, para se obter a melhor abordagem de BD para utilização no problema proposto.

\subsection{O modelo de BD relacional}

O modelo entidade-relacionamento criado para a confecção do BD relacional é representado na Figura 1. Note que são identificados quatro entidades principais: Artigo, Autor, Evento e Instituição, e três relacionamentos. As características das entidades que foram recuperadas no estudo de caso são representadas por atributos. A definição das tabelas e dos relacionamentos que foram inseridos no BD é baseada nos dados recuperados nas trilhas técnicas dos simpósios.

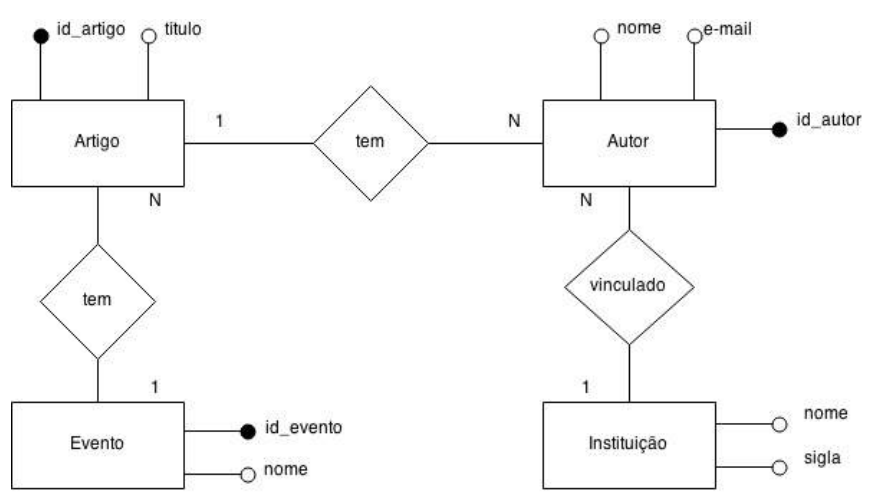

Figura 1: Modelo entidade-relacionamento.

\subsection{O modelo de BD orientado a grafos}

Após a criação do banco de dados em modelo relacional, foi criado um BD orientado a grafos, que recebeu a mesma carga de dados do modelo relacional. Por se tratar de outra abordagem de BD o modelo foi construído de forma distinta, onde as chaves e identificadores tiveram de sofrer alterações para atender as necessidades desse modelo de BD.

O modelo criado para a base de dados orientada a grafos utilizada neste trabalho é identificado na Figura 2. No modelo orientado a grafos, as entidades que antes eram representadas em tabelas no modelo relacional são representadas em vértices e os relacionamentos em arestas, sendo os atributos identificados no modelo relacional representados como propriedades no modelo orientado a grafos.

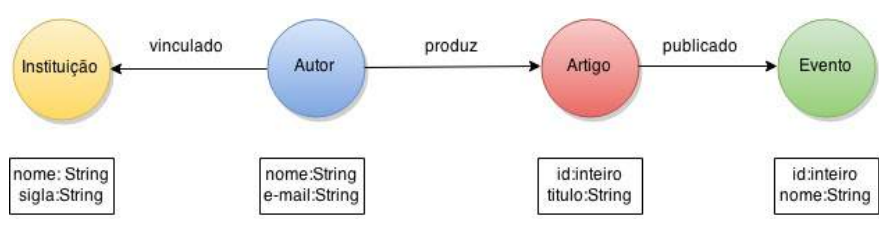

Figura 2: Modelo orientado a grafos. 

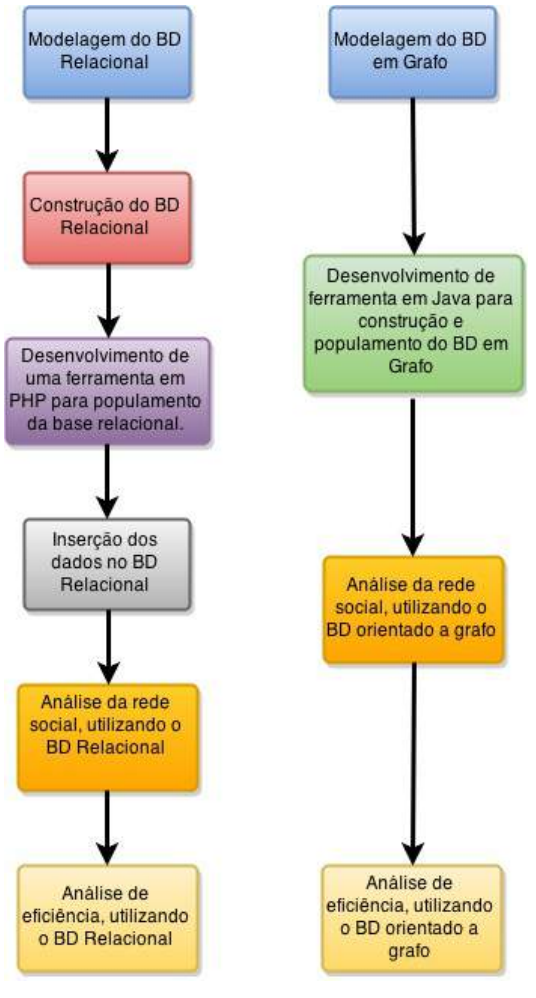

Figura 3: Ordem de execução dos passos empregados na metodologia deste trabalho.

\subsection{Detalhamento do desenvolvimento}

Os passos do desenvolvimento deste trabalho são representados diagramaticamente na Figura 3, onde os passos de desenvolvimento estão representados em paralelo para as duas abordagens, sendo a relacional a da esquerda e a em grafos da direita.

Primeiramente, os modelos de BD foram criados paralelamente, sendo representados nas Figuras 1 e 2.

Após a criação dos modelos de BD utilizados neste trabalho, iniciou-se a etapa de criação do BD Relacional, sendo criado manualmente no SGBD MySQL.

Em seguida, foram feitas as inserções das informações no BD Relacional. Os dados inseridos são referentes as edições de 2006 a 2011 e foram importados do estudo realizado por [1]. Os dados importados dessa pesquisa estavam disponíveis para importação apenas na linguagem SQL, tornando-se necessário o início do populamento ser no modelo relacional criado. Após a importação desses dados, os dados referentes às edições de 2005, 2012, 2013 e 2014, foram inseridos manualmente no BD relacional, com o auxílio de um sistema em PHP, facilitando as inserções e consultas no BD.

A etapa de criação e inserção dos dados no BD Relacional foi executada somente após essas etapas serem executadas no BD Relacional. Com o término da criação e inserções no BD Relacional, a mesma etapa de criação e inserções manuais deveria ser repetida para o banco de dados orientado a grafos, porém por se tornar um processo repetitivo, foi criado um script em Java que criou o BD Orientado a Grafos e exportou os dados inseridos no BD relacional para o BD Orientado a Grafos.

O script se conectava ao BD Relacional por meio de co-
Tabela 1: Total de artigos e autores por edição do SBSI.

\begin{tabular}{l|l|l} 
EDIÇÃO & ARTIGOS & AUTORES \\
\hline 2005 & 33 & 101 \\
2006 & 39 & 109 \\
2008 & 39 & 108 \\
2009 & 29 & 96 \\
2010 & 40 & 114 \\
2011 & 32 & 88 \\
2012 & 76 & 220 \\
2013 & 85 & 240 \\
2014 & 63 & 187 \\
\hline TOTAL & 436 & 1019
\end{tabular}

nectores MySQL e ao BD Orientado a Grafos por meio de uma biblioteca [11] que o Neo4j disponibiliza para projetos em Java, assim tornando-se possível consultas e inserções em ambiente Java.

A mesma base de dados foi gerada para os dois modelos de BD estudados. Com as bases de dados populadas com as informações foi feita a análise das informações da rede social científica utilizando juntamente as duas abordagens de $\mathrm{BD}$, e após foi executada a análise de performance das abordagens de BD. As etapas das análises são detalhadas na Seção 5 .

\section{ANÁLISE DOS RESULTADOS}

Nesta seção são apresentadas as questões levantadas na Seção 1 e as análises e resultados comparativos entre as diferentes abordagens de BD adotadas na pesquisa.

\subsection{Análise das informações da comunidade de SI}

Com as informações das edições SBSI 2005 a 2014 armazenadas em banco de dados, foram feitas algumas consultas e análises que possibilitaram verificar algumas características da rede social utilizada. Algumas dessas informações podem ser visualizadas na Tabela 1 e nas Figuras 4 a 6 .

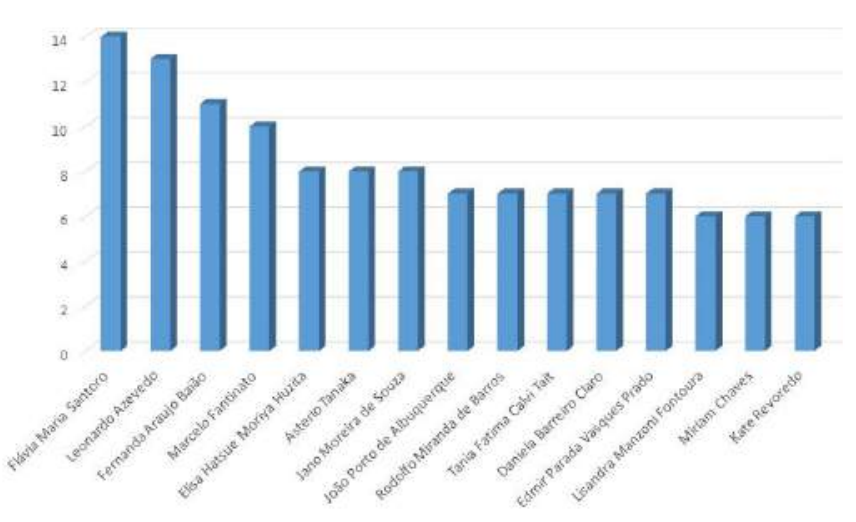

Figura 4: Autores com maior frequência de publicação nas edições SBSI.

Com a análise das informações da comunidade estudada, foi possível identificar algumas características da rede social e responder algumas questões definidas para a execução 


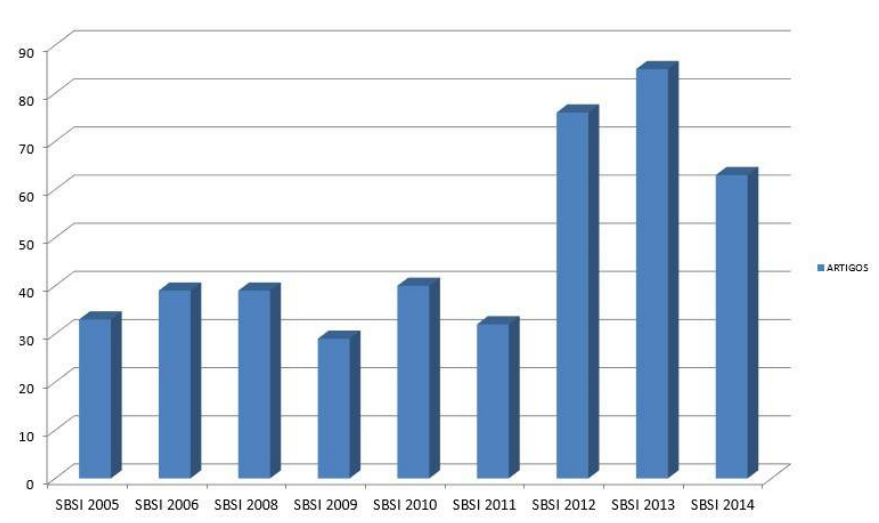

Figura 5: Quantidade de artigos publicados nas Trilhas Técnicas por cada edição.

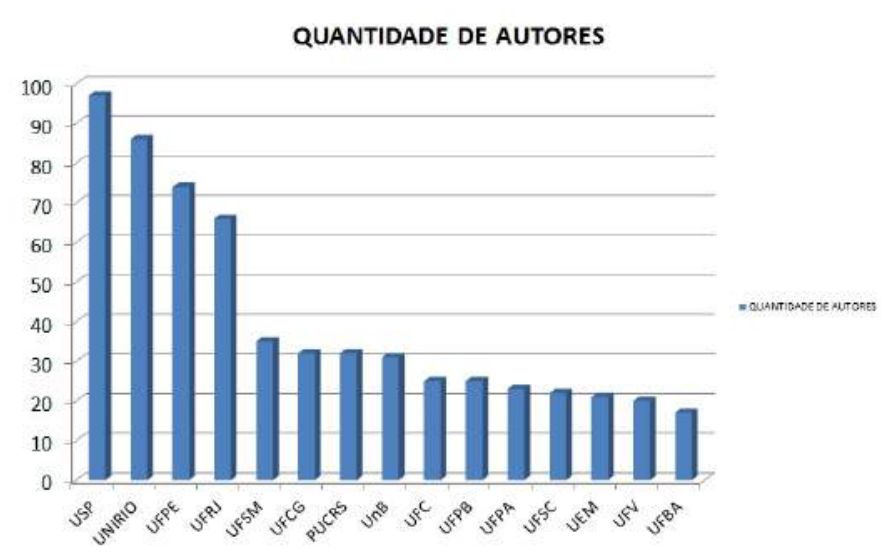

Figura 6: As 15 instituições de vinculação dos autores com maior frequência de publicação nas edições SBSI.

deste trabalho. Foi identificado qual o autor que mais publicou em todas as edições do Simpósio, quantos artigos foram publicados em cada edição e qual a instituição ofereceu mais autores para publicação.

Na Tabela 1 é possível identificar o total de artigos publicados e a quantidade de autores que publicaram em cada edição do SBSI. Como um mesmo autor pode publicar em edições distintas do SBSI, a soma da quantidade de autores por edição não é o total de autores que já publicaram no SBSI.

Também é possível consultar informações específicas. A Figura 7 mostra o grafo gerado com as informações de publicação para um determinado autor. A consulta retornou quais os artigos uma determinada autor publicou e em quais edições do SBSI foram publicados.

Com esta análise também é possível verificar quais os autores já publicaram em co-autoria com um determinado autor. Na Figura 8 é possível ver uma representação em grafo dos autores que já publicaram em co-autoria com determinado autor, sendo possível a visualização dos artigos resultantes dessas co-autorias.

O conceito de profundidade que é utilizado para descrever a consulta é o mesmo conceito de profundidade de árvores

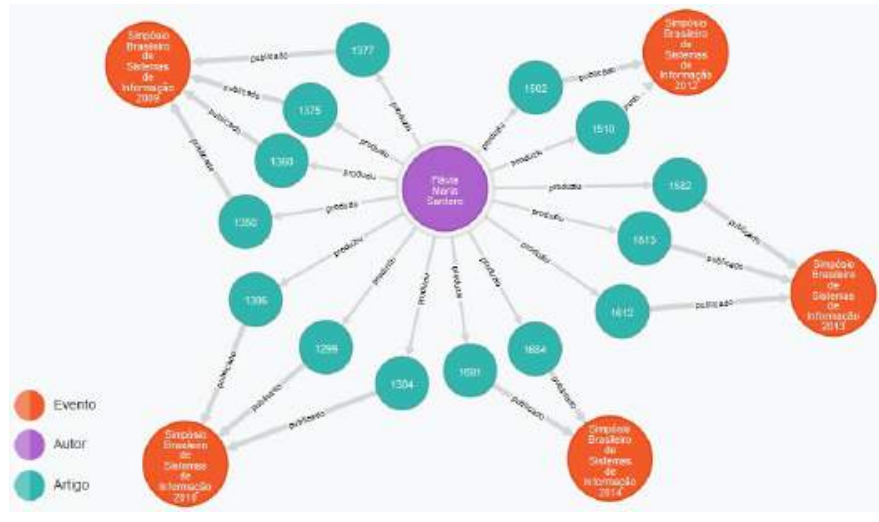

Figura 7: Representação em grafo das publicacões de um autor específico.

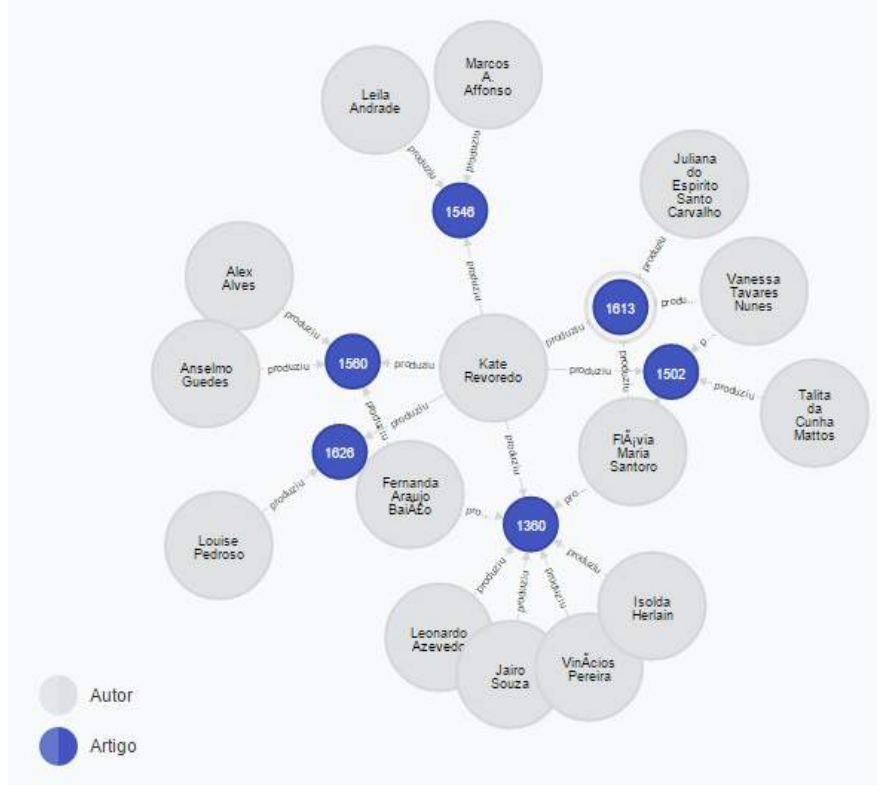

Figura 8: Representação em grafo de co-autores de determinado autor.

definido por [2]. A consulta de co-autoria é uma consulta em profundidade, pois a consulta verifica o nó ou a entidade autor mais de uma vez. De acordo com [2], toda arvóre pode ser representada por um grafo. Como a consulta de co-autoria é iniciada em um nó de autor que busca outro nó autor, podemos representá-la em uma estrutura de dados de árvore.

A consulta utilizada na Figura 8, é uma consulta de profundidade 1, pois a partir de um Autor $X$ o retorno da consulta é outro Autor $Y$. Se a consulta retornar um Autor $Z$ a partir de um Autor $Y$ resultante de outro Autor $X$, a consulta teria profundidade 2. Assim, pode-se definir que $\mathrm{P}=$ $\mathrm{Q}$ - 1, onde $\mathrm{P}$ é a profundidade da consulta, e Q é quantidade de vezes que a consulta percorre a entidade autor.

\subsection{Análise de performance dos BD's}

Com a análise dos dados da comunidade de SI que publicaram nas diversas edições do SBSI executada, tornou-se possível realizar a análise dos BD's e assim verificar qual 
Tabela 2: Consulta que retorna a quantidade de artigos publicados em cada edição.

\begin{tabular}{|l|}
\hline BD Relacional \\
\hline SELECT count $\left({ }^{*}\right)$, e.NOME \\
FROM artigos a, eventos e \\
where a.FK_EVENTO = e.ID_EVENTO \\
group by e.NOME; \\
\hline BD Orientado a Grafos \\
\hline MATCH (a:Artigo)-[:publicado]-> (e:Evento) \\
RETURN e.ID, count(a.ID) ORDER BY e.ID; \\
\hline
\end{tabular}

Tabela 3: Consulta que retorna todos os autores que publicaram em co-autoria com determinado autor.

\begin{tabular}{|l|}
\hline BD Relacional \\
SELECT distinct t.nome \\
FROM artigos a, autores $t$, autores_artigos ar \\
WHERE a.id_artigo = ar.id_artigo \\
and t.id_autor = ar.id_autor \\
and a.id_artigo in (select a.id_artigo from \\
artigos a, autores t, autores_artigos ar \\
WHERE a.id_artigo = ar.id_artigo and \\
t.id_autor =ar.id_autor \\
and t.nome = 'Fulano de Cicrano'); \\
\hline BD Orientado a Grafos \\
\hline MATCH (a:Autor)-[:produziu]->(b:Artigo) \\
$<-[:$ produziu]-(d:Autor) \\
WHERE a.Nome = 'Fulano de Cicrano' \\
RETURN DISTINCT d.Nome;
\end{tabular}

abordagem de $\mathrm{BD}$ nos oferece de forma mais eficiente os dados recuperados para análise. Cada consulta foi realizadas 10 vezes recuperando o tempo de cada execução. A ferramenta $M y S Q L$ Workbench 6.2 foi utilizada para a realizar as consultas e recuperar os tempos de execução das consultas no BD Relacional. Para execução das consultas e recuperação dos tempos de execução do BD orientado a grafos, foi utilizada a ferramenta Neo4j Community Edition. A média dessas métricas foi considerada como o tempo de cada consulta, sendo utilizado para realizar a análise de performance dos BD's.

Para análise de eficiência dos BD's foram executadas três consultas de profundidade 0 , uma consulta de profundidade 1 , uma consulta de profundidade 2 , uma consulta de profundidade 3 e uma consulta de profundidade 4 . As Tabelas 2 e 3 e mostram um exemplo de uma consulta de profundidade 0 e um exemplo de profundidade 1 respectivamente, para as duas abordagens de BD. A Tabela 4 mostra as médias das consultas para cada abordagem de BD.

$\mathrm{Na}$ análise comparativa da eficiência de duas abordagens de $\mathrm{BD}$, foi possível verificar que para as consultas de profundidade 0 e 1 , o BD relacional tem uma pequena vantagem de eficiência. Quando a consulta se estende para profundidade 2 os dois tipos de BD têm basicamente o mesmo tempo de eficiência. A partir da profundidade 3 o BD orientado a grafos tem um menor tempo de consulta, com uma concreta vantagem no tempo de consulta na profundidade 4 , podendo ser visualizado na Figura 9.

\section{CONCLUSÕES E TRABALHOS FUTUROS}

Este trabalho apresentou uma análise da comunidade de
Tabela 4: Tempo em milissegundos da execução das consultas em cada abordagem de banco de dados.

\begin{tabular}{l|l|l} 
Profundidade & Relacional (ms) & Grafos (ms) \\
\hline 0 & 0,007 & 0,024 \\
0 & 0,028 & 0,046 \\
0 & 0,051 & 0,068 \\
1 & 0,028 & 0,062 \\
2 & 0,078 & 0,080 \\
3 & 1,568 & 0,979 \\
4 & 23,762 & 10,207 \\
\hline
\end{tabular}

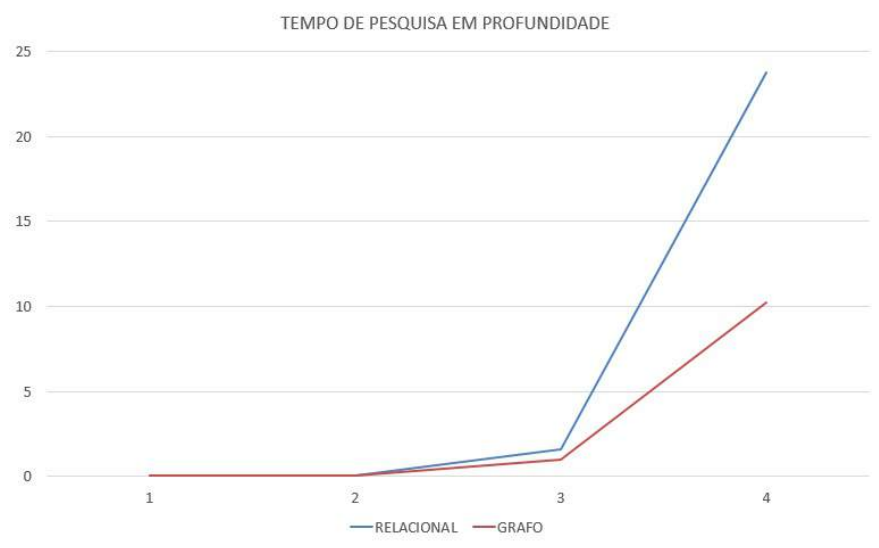

Figura 9: Tempo de consulta em profundidade das duas abordagens de banco de dados.

SI com foco nas edições de 2005 a 2014 do SBSI gerada pelas informações dos artigos publicados na trilha principal e uma análise comparativa entre duas abordagens de BD, sendo uma relacional e a outra orientada a grafos.

Os objetivos definidos na Seção 1 foram alcançados, pois as questões levantadas foram respondidas com a análise da Comunidade de SI realizada.

Sobre o estudo para se definir a melhor abordagem de banco de dados a se utilizar em uma rede social científica, a realização da análise de eficiência nos ajudou a levantar características e informações do funcionamento dessas abordagens distintas de BD neste tipo de rede social.

Para o BD orientado a grafos, foi verificado que para redes onde é necessário realizar consultas de grandes profundidades e que necessite do cruzamento de dados de uma mesma entidade ou tipo de nó, como autor, é uma melhor alternativa em comparação com o modelo relacional, pois foi verificado que o tempo de consulta é menor. Pois no BD relacional esta consulta necessita de várias junções entre as entidades, diferente do BD orientado a grafos que utilizam os relacionamentos entre os grafos para realizar este tipo de consulta.

Com essa base de dados disponível para a comunidade, várias consultas poderão ser executadas, assim servindo para recuperar informações que podem ser necessárias para auxiliar a comunidade científica responsável pelas publicações no SBSI, servindo de base também para se ter conhecimento do panorama dos trabalhos relacionados à SI no Brasil.

A proposta de trabalhos futuros é a aplicação deste estudo em redes sociais de maior escala, juntamente com a disponibilização de uma ferramenta para a pesquisa das in- 
formações levantadas nessa rede social científica do SBSI, com visualização das estruturas em grafos, resultantes das consultas representadas graficamente e opção de inserção de informações das futuras edições do SBSI.

\section{REFERÊNCIAS}

[1] E. A. de Oliveira and V. M. F. Dias. Redes sociais do sbsi e o corte de vértices como base para identificar atores importantes na coesão de grupos de pesquisa. Simpósio Brasileiro de Sistemas de Informação 2012 (SBSI), 2012.

[2] M. J. A. Aaron Ai Tenenbaum, Yedidyah Langsam. Data Structures Using C. McGraw-Hill, Inc., 1989.

[3] C. Alberto Heuser. Projeto de Banco de dados: Uma visão prática. Editora Sagra Luzzato, 1998.

[4] F. E. Codd. Relational model of data for large shared data banks. IBM Research Report, San Jose, California, 13(6):377-387, 1970.

[5] D. J. Cook and L. B. Holder. Graph-based data mining. IEEE Intelligent Systems, 15:32-41, 2000.

[6] E. A. de Oliveira. Sobre a Colaboração na Comunidade de Sistemas de Informação. Master's thesis, Universidade Federal do Estado do Rio de Janeiro, Rio de Janeiro, Brasil, 2012.

[7] A. M. de Souza, E. Prado, V. Sun, and M. Fantinato. Critérios para seleção de sgbd nosql: o ponto de vista de especialistas com base na literatura. Simpósio Brasileiro de Sistemas de Informação 2014 (SBSI), 2014.

[8] K. Finley. 5 graph databases to consider, Abril de 2011. http://readwrite.com/2011/04/20/ 5-graph-databases-to-consider, Acessado em 3 de Fevereiro de 2015.

[9] F. S. Foundation. The gnu general public license v3.0 gnu project - free software foundation. http://www.gnu.org/copyleft/gpl.html, Acessado em 3 de Fevereiro de 2015.

[10] F. Inc. Allegrograph rdfstore web 3.0's database. http://franz.com/agraph/allegrograph/, Acessado em 3 de Fevereiro de 2015.

[11] N. T. Inc. Include neo4j in your project - the neo4j manual v2.2.1. http://neo4j.com/docs/stable/ tutorials-java-embedded-setup.html, Acessado em 3 de Fevereiro de 2015.

[12] N. T. Inc. Neo4j graph database. http://neo4j.com/product/, Acessado em 3 de Fevereiro de 2015.

[13] O. inc. Infinitegraph | distributed graph database. http://www.objectivity.com/infinitegraph, Acessado em 3 de Fevereiro de 2015.

[14] F. N. R. Machado and M. P. de Abreu. Projeto de Banco de dados: Uma visão prática. Editora Erica, 2009.

[15] J. A. N. G. Manzano. MySQL 5.1 Interativo - Guia Básico de Orientação e Desenvolvimento). Editora Erica.

[16] J. J. Miller. Graph database applications and concepts with neo4j. In Proceedings of the Southern Association for Information Systems Conference, Atlanta, GA, USA March 23rd-24th, 2013.

[17] A. B. M. Moniruzzaman and S. A. Hossain. Nosql database: New era of databases for big data analytics
- classification, characteristics and comparison. CoRR, abs/1307.0191, 2013.

[18] I. Robinson, J. Webber, and E. Eifrem. Graph Databases. O'Reilly Media, Incorporated, 2013.

[19] Ł. Warchał. Using neo4j graph database in social network analysis. Studia Informatica, 33(2A):271-279, 2012 . 
XI Brazilian Symposium on Information System, Goiânia, GO, May 26-29, 2015. 